\title{
Of Money and Morals - The Contingent Effect of Monetary Incentives in Peer-to-Peer Volunteer Computing
}

\author{
Janick Edinger Laura Marie Edinger-Schons Dominik Schäfer Aleksander Stelmaszczyk Christian Becker \\ University of Mannheim \\ $\underline{\text { janick.edinger@ }} \quad \frac{\text { schons@ }}{\text { nni-mannheim.de }} \quad \frac{\text { dominik.schaefer@ }}{\text { bwhi-mannheim.de }} \frac{\text { aleksander.stelmaszczyk@ }}{\text { uni-mannheim.de }} \frac{\text { christian.becker@ }}{\text { uni-mannheim.de }}$ \\ uni-mannheim.de bwl.uni-mannheim.de uni-mannheim.de uni-mannheim.de uni-mannheim.de
}

\begin{abstract}
Driven by technological advances, the recent trend of the sharing economy has brought up multiple globally successful companies, a disruption of business models, and presumably more sustainable alternatives to traditional resource allocation and consumption. Instead of depending only on professional companies, people increasingly share their resources in peer-to-peer networks. In volunteer computing systems, for example, device owners share their spare computational resources with other users. Despite the success stories in other businesses in the sharing economy, however, the popularity of such peer-to-peer computing systems has remained limited. The authors focus on the perspective of resource providers and develop a framework of the effectiveness of monetary incentives to motivate resource providers in volunteer computing. Drawing from Relational Models Theory and Motivation Crowding Theory, the framework proposes a three-way interaction between monetary incentives, social relationships, i.e., sharing with anonymous users versus with friends, and the individual predisposition of the user, i.e., their moral identity centrality. Informed by a preliminary survey, a between subjects experiment tests the propositions and delivers full support for the hypothesized contingencies. Monetary incentives can enhance the intrinsic motivation to share resources when sharing takes place amongst anonymous users. However, paying monetary rewards can disrupt motivation when sharing takes place among friends, especially when users have a high moral identity centrality. The authors discuss their result in the light of their conceptual and practical implications.
\end{abstract}

\section{Introduction}

The sharing economy has become a promising alternative to traditional consumption behavior [1]. Technological advances and the omnipresence of a fast
Internet connection have made sharing resources not only easy but also massively scalable. In examples like Uber or AirBnB resource providers simply need to register their resources or services online and within only a few minutes their offer is visible for millions of people. It is, thus, safe to say that information and communications technology is the key enabler for the sharing economy. Besides accommodations and rides, computational power is a resource that can be shared on a global scale in a peer-to-peer (P2P) fashion. With the growing demand of processing power on the one side and the increasing number of computing devices on the other side, computational resource sharing is the logical consequence. The chance of exploiting the free computational power was seized decades ago by projects like Condor [2] or Great Internet Mersenne Prime Search (GIMPS) [3]. Other classic examples include the BOINC middleware [4] which supports numerous scientific projects based on volunteer computing. Mass participation of motivated users in the sharing scheme is a crucial requirement for building a large-scale distributed system. While computational resource sharing for scientific projects has already been successful for many years, peer-to-peer volunteer computing systems have never achieved a comparable popularity. Thus, in this paper, we shed light on how resource owners can be incentivized to share their computational power. More specifically, we examine the effect on monetary incentives on the willingness to participate.

The role of monetary incentives for sharing is not yet well understood. Haas et al. [5] suggest that different types of social relationships require different incentives for sharing in P2P computing. However, they do not provide empirical evidence. Extant research has revealed that extrinsic rewards can lead to both a crowding-in as well as a crowding-out of the intrinsic motivation to engage in prosocial behaviors such as sharing. Do monetary incentives increase or decrease resource providers' willingness to participate in peer-to-peer volunteer computing systems? Drawing 
from relational models theory $([6,7])$ and motivation crowding theory $([8,9])$ we derive a conceptual framework in which we propose that the effect of monetary incentives in volunteer computing systems depends on the relationships between resource providers and consumers. Both theories play important roles in the sharing economy as sharing happens among both strangers and friends and different exchange mechanisms are applied. The interplay of relationship types and exchange mechanisms might thereby lead to crowding out effects. Further, we argue that the personal predisposition of the resource provider (i.e., their moral identity centrality) might have an impact on the willingness to share resources. To test our hypotheses we conduct a $2 \times 2 \times 3$ between subjects scenario experiment in which we manipulate whether respondents receive a monetary reward, whether they share resources with anonymous users or friends, and whether the system uses gamification elements. Results fully support our theorizing. We find a three-way interaction between monetary incentive, type of relationship, and respondents' moral identity centrality. Specifically, whereas in case of sharing with anonymous users, the effect of monetary incentives on willingness to participate is significantly positive only for those respondents high on moral identity centrality, for the case of sharing with friends we find the opposite. Here, the effect of monetary incentives is positive for low levels of moral identity centrality but turns negative for high levels. We do not find significant effects of the use of gamification elements in our setting. Our results have implications for academic theory and for the design of platforms for sharing of computational resources alike. In terms of conceptual contributions, our results contribute to the literature streams on collaborative consumption and production on P2P platforms in the sharing economy, relational models theory and market mechanisms in the sharing economy, motivation crowding theory and the role of intrinsic and extrinsic motivation in the sharing economy, and moral identity as a predictor of sharing.

\section{Volunteer Computing}

Modern offloading mechanisms, virtualization techniques, and the rapid growth of the global networking infrastructure make it possible to seamlessly transfer workload to remote machines. This concept is known as task offloading and facilitates computational resource sharing where workload is exchanged between geographically distributed computing devices. Resource providers offer their idle computing capacities to resource consumers that run computationally intensive applications.Commercial resource providers such as Amazon EC2 provide computing resources in a pay-per-usage fashion. This business model is well suited for companies that shift their computational workload from on-premise resources to cloud instances. As the resource demand of recent applications like rendering virtual reality, processing video streams, or running artificial intelligence algorithms exceeds the capabilities of user-owned devices, computation offloading becomes important also for non-commercial users. Thus, private users themselves become resource consumers. Further, the amount of computational resources is increasing and the processing power of end-user devices often remains unused. Device owners can share their resources and become resource providers for others in volunteer computing systems, such as OurGrid [10], SocialCloud [11], Social Cloud Computing [12], and Cloud@home [13]). These P2P systems can either have an underlying economic model or enable sharing resources for free.

\section{Determinants of Sharing Computational Resources}

One of the most discussed topics in social distributed computing is the question why users would share their resources with others. In the literature, we have identified three key factors that have an impact on the users' willingness to participate. These factors are motives, social relationships, and obstacles.

\subsection{Motives}

In [14], Ryan and Deci summarize a distinction between types of motivation, based on their Self-Determination Theory: The most basic distinction is between intrinsic motivation, which refers to doing something because it is inherently interesting or enjoyable, and extrinsic motivation, which refers to doing something because it leads to a separable outcome. [14, p. 55] In the following, we discuss different types of motivation from the rather intrinsic to more extrinsic motivations.

Altruism - The concept of altruism is often referred to as a basic motivation in crowd sourcing scenarios. Altruism can be considered the most intrinsic type of motivation. Altruistic users are willing to share their resources for free. The mere activity of sharing and the feeling of warm glow are inherently enjoyable or rewarding. This can for instance be the case for some scientific projects in which users support the goals of the project and are intrinsically motivated to move the idea forward.

Gamification - Starting from 2011, the idea 
of gamification became popular [15], meaning the embedding of game elements into non-game contexts to make these experiences enjoyable. In volunteer computing, gamification can be found when contribution is rewarded with credits that can be compared with those of other participants. Even though these rewards do not have an actual monetary value, they may increase the motivation to participate and introduce competition between users.

Reputation - Reputation becomes relevant as a motivation when achievements are connected to real persons, e.g., when users of volunteer computing have the possibility to share their results in social media networks. In [16], Ariely et al. show that people have a strong desire to be seen by others as doing good. Communicating that they have provided computational resources, e.g., for a scientific project, can be a signal that individuals can use to build up a reputation for doing good.

Reciprocity - Sharing can be performed in a reciprocal way where resource consumers share their own resources in the future and resource providers receive some kind of credit that they can later trade for computation. This protocol can be implemented in multiple one-to-one relationships where the resource usage between two users needs to balance out. A more flexible solution is to introduce a credit system which allows to trade computation between multiple users.

Virtual Currency - Even though reciprocity can be one way to solve the free-rider problem, it is limited to scenarios in which the exact same resource is traded. This may lead to a situation in which a user who mainly wants to use computational resources from other users but cannot provide these resources in the future will be excluded from the system. In turn, users that own powerful resources accumulate credits that they will not need to spend as they are never reliant on remote computational resources. A virtual currency can help to solve this problem as it might enable users to trade computational power for other resources such as bandwidth or storage.

Monetary - Finally, users in P2P systems can even use real money in transactions. When users pay for using remote resources, they do not have the obligation to share resources themselves. Instead, each resource has its price which can be either fixed, variable, or can be negotiated between resource consumer and provider.

\subsection{Relationships to Consumers}

A fact that is often neglected in distributed computing systems is that devices are owned by private users and that these users might have social relationships. In the following, we categorize different relationships and discuss what impact they may have on the willingness to share resources.

Anonymous - In many distributed computing systems, resource consumers and providers do not know each other. Resource consumers have no control over the decision on which device their task is executed and, in turn, resource providers cannot decide who runs which task on their machines.

Project-based - In volunteer computing for scientific projects, users can decide to which project they want to contribute. Therefore, the project owners advertise their undertaking and explain how this project can be beneficial for the public. Resource providers then select one or multiple projects.

Social Networks - Friendship relations in social networks are often based on real-world relationships [17]. Resource owners might rather be willing to provide their computational power to their friends and family than to strangers. Thus, the virtual relationships of social networks can be leveraged to match resource consumers and providers. Besides leveraging existing social networks, there can also be networks that are designed and implemented for the purpose of resource sharing. Given that there is a certain level of trust between friends in social networks and issues such as the free-rider problem or privacy concerns become less relevant.

Personal Contact - Tasks can be offloaded in one-to-one relationships where a provider explicitly grants another user access to resources. One possible scenario is sharing between people gathered in one room or in proximity. This solution borrows from the concept of opportunistic computing [18]. The level of trust can be considered very high in these systems.

\subsection{Obstacles}

Obstacles are reasons that would discourage users to participate in volunteer computing systems. We discuss several of these reasons in the following.

Cost - Users might simply not want to share resources as the additional workload would require more energy and thus result in higher costs. Especially when there is no compensation, sharing resources is comparable to donating money.

Security - Even though many distributed computing systems are sandboxed and are not considered a security threat, many people have mixed feelings about running unknown code on their devices.

Lack of Motivation - Users might not see any reason why participating in distributed computing 
systems could be beneficial for them. They might never have experienced situations in which they would have required more computational power and thus do not expect any benefit from a resource sharing system. Further, the amount of money that they could earn by renting out their resources in commercial systems might not be sufficiently incentivizing.

Effort to Participate - Sharing resources requires at least some manual setup. Users need to install software that allows to share resources and register an account. In volunteer computing, users also have to select one or more projects they would like to contribute to. When resources of mobile devices are shared, device owners might need to charge these devices more often which leads to additional effort.

Device Slow-Down - As each device has a finite amount of processing power, sharing resources might result in a slower execution of user applications. Thus, users might experience a slow-down of their own applications due to the execution of other people's tasks.

\section{Pre-study}

In a first step, we decided to conduct a pre-study amongst potential users to elicit their awareness of possibilities to share computational resources, their motives and willingness to participate in such sharing systems depending on incentives and social relationships, and potential obstacles that would hinder them from participating. We recruited our participants via Amazon's Mechanical Turk. The sample comprises 208 US-based respondents. The mean age in the sample is 37.87 years and $49 \%$ of the respondents are female. The respondents received $.20 \$$ as compensation for participation in the survey that took on average approximately 6 minutes.

To establish an equal understanding of what sharing of computational resources means, we included an explanatory text at the beginning of the questionnaire ${ }^{1}$.

First, we asked respondents whether they owned a smartphone, which computational tasks they typically performed on their devices, and whether they feel that limited computational power is a problem for them.

Although respondents on average did not agree that limited computational resources pose a problem for them, they agreed that they have experienced their smartphone battery to drain faster due to a computational intensive application and have felt that an application might be faster if their phone had more computational resources.

Further, to ensure that all respondents understand that sharing can work in both directions, i.e.,

\footnotetext{
${ }^{1}$ Experimental material will be provided by the authors on demand
}

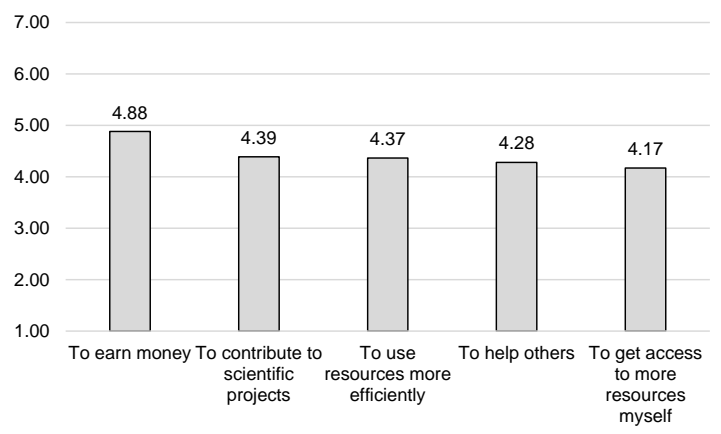

Figure 1. In general, what would be your motivation to participate in such a system? $(1=1$ do not agree at all to $7=$ I fully agree)

respondents can use other users' computational resources but can just as well be providers of computational resources, we included an explanatory text.

Next, we asked respondents whether they are aware of the possibility to share computational resources and whether they have made past experiences with sharing. $50 \%$ of the respondents reported to be aware of the possibility to share computational resources with others and $26.9 \%$ of these respondents have made experiences with sharing of computational resources. Asked for which kind of experience they have made, most of these respondents report to have participated in SETI@ @ome and 80\% explained that the experience with sharing of computational resources has been a positive one. Moreover, we were interested in respondents' future willingness to share computational resources with others. Specifically, we asked them about their general willingness to share computational resources with other users ('Would you be willing to share your computational resources with others? I would share my resources with others.', $1=\mathrm{I}$ do not agree at all to $7=\mathrm{I}$ fully agree, mean value $=3.91$ ), their willingness to share depending on social relationships and incentives, and potential obstacles to sharing.

We further directly asked respondents what their motivation would be to participate in a system to share computational resources. Here, results reveal the highest agreement for 'to earn money'. The more altruistic motives 'to contribute to scientific projects', 'to use resources more efficiently', and 'to help others' rank second, third, and fourth, while 'to get access to more resources myself' received the least agreement (compare Figure 1).

We were also interested to know which obstacles would hinder respondents from participating in sharing resources with other users. In a descriptive analysis 


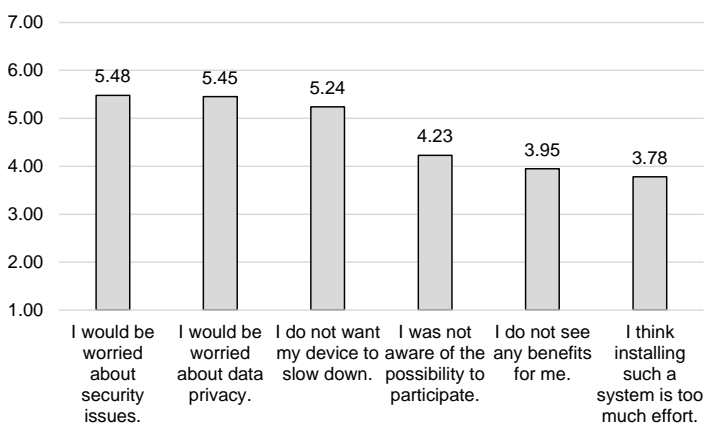

Figure 2. Which of the following reasons could hinder you from sharing your resources? $(1=1$ do not agree at all to $7=$ I fully agree)

of the data, the strongest agreement can be found for security issues, data privacy, and worries that the own device could slow down (compare Figure 2).

In terms of social relationships, we were interested to know whether respondents feel that varying compensation mechanisms would be appropriate for different groups of users. To introduce the possibility of varying compensation mechanisms, we explained to them: 'Imagine a system that allows you to safely share your computational resources with others. Now, imagine a system in which device owners can be compensated for sharing their resources. They can be compensated in multiple ways: i) They could share their resources for free, which means that they do not receive a compensation. ii) They could receive a compensation to cover their energy costs. iii) They could receive a compensation to make a profit. Which compensation would you demand from the following users for sharing your resources?'

With friends and family, $67.8 \%$ of respondents would share computational resources for free. Interestingly, in case of scientific projects, for-profit organizations, and anonymous private users, the majority of respondents $(43.7 \%, 58.3 \%$, and $41.1 \%)$ would want the exchange to be profitable for them. In case of non-profit organizations, the majority $(39.8 \%)$ would just expect their costs to be covered. Further, in case of scientific projects and non-profit organizations, a considerable share of respondents would be willing to share their computational resources for free $(10.7 \%$ and $14.1 \%$ ) whereas this is not the case for for-profit organizations and anonymous private users (compare Figure 3).

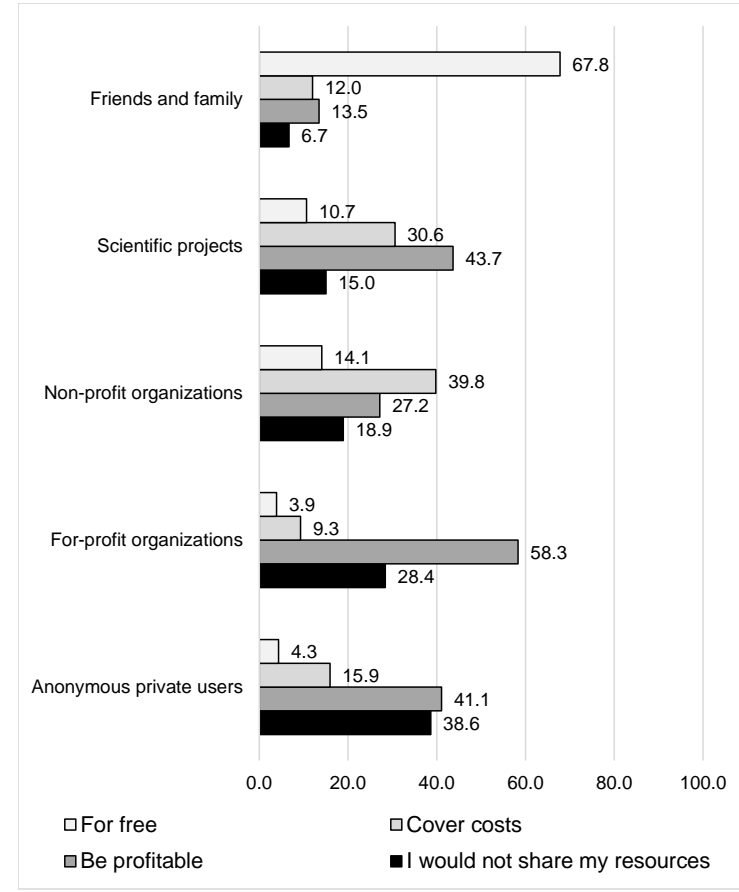

Figure 3. Which compensation would you demand from the following users for sharing your resources? For each of the four user groups participants could decide whether they would share their resources for any kind of compensation or whether they would not share their at all.

\section{Empirical Study}

The pre-study revealed some interesting insights which informed our following work in which we focus on $\mathrm{P} 2 \mathrm{P}$ systems. Interestingly, monetary incentives seem to play an important role, given that respondents rate earning money as the most important motivation to engage in resource sharing. However, the type of social relationships can obviously not be neglected as it seems to determine the compensation that users expect from their peers. In the following, we will derive a framework of the effectiveness of monetary incentives in P2P volunteer computing networks and test it in a between subjects experiment.

\subsection{Hypotheses development}

Extrinsic incentives have been found to be effective to enhance prosocial behaviors such as resource sharing $[19,16]$.

However, there is a considerably large literature stream which has revealed that extrinsic incentives may lead to both, a crowding-in or a crowding-out of the intrinsic motivation to engage in prosocial behaviors 
$[8,9]$. In other words, monetary rewards can strengthen or harm the intrinsic motivation to do good deeds.

We propose that the question whether monetary incentives enhance or reduce the willingness to participate in volunteer computing depends on the type of relationship between resource providers and consumers. We draw from Relational Models Theory $[6,7]$ to make this argument. Heyman and Ariely (2004) explain that humans categorize interactions as either social or monetary and that their reactions to monetary incentives depend on this categorization. If the relationship is categorized as social, providing a monetary incentive may harm accepted norms of behavior. To illustrate, imagine the following situation: If your best friend asks you to help her with her house move, you would most probably not consider to charge her an hourly fee.

In line with this literature, we expect monetary incentives to increase the willingness to participate in volunteer computing if the consumer group is made up of anonymous users. This is due to the fact that the exchange will be categorized as a monetary transaction and monetary incentives will be considered appropriate. On the contrary, we expect monetary incentives to reduce the willingness to participate in volunteer computing if sharing takes place amongst friends. In this case, the exchange resembles a social interaction and receiving a monetary reward would harm social norms of friendship (e.g., you should not financially benefit from your friends). Thus, in summary, we expect monetary rewards to lead to a crowding-in of the motivation to participate for anonymous private users and to a crowding-out for sharing amongst friends.

Finally, over and above the interaction between compensation and relationship type, we argue that these effects depend on the personal predisposition of the individual user. Specifically, we expect the users' centrality of moral identity [20] to be a decisive factor. The centrality of moral identity is conceptualized as "the cognitive schema a person holds about his or her moral character" [p.124][21]. It is stored in a person's memory as a complex knowledge structure consisting of moral values, goals, traits, and behavioral scripts ([20, 22]). These knowledge structures are assumed to be acquired through life experiences and therefore to differ across individuals [23]. For people whose moral identity occupies greater centrality within the self-concept, being a moral person is more self-definitional compared to other identities [24].

We expect that individual differences in the centrality of a person's moral identity will play a key role in determining their reactions to monetary incentives in the context of volunteer computing.
Individuals with a high centrality of moral identity will strive for consistency with their ideal of being a moral person. This means that they will try to adhere to accepted social norms of behavior, as, e.g., that you should not financially benefit from your friends. For these individuals, a monetary incentive for sharing resources with friends will likely lead to a reduction in the willingness to participate (i.e., crowding-out). In a monetary interaction, however, the monetary incentive will strengthen these users' intention to participate (i.e., crowding-in). Individuals with a low centrality of moral identity on the other hand, will put less value on following such rules of social interactions. For these individuals, a monetary incentive will most probably have a positive effect and strengthen their willingness to participate independent of the type of relationship. To summarize, we expect that in case of anonymous private users, monetary incentives will lead to a higher motivation to share for participants with a high moral identity than for those with a low moral identity. Conversely, we argue that that amongst friends, monetary incentives will lead to a lower motivation to share for participants with a high moral identity than for those with a low moral identity. We formally hypothesize:

H1: monetary incentives enhance the willingness to participate in volunteer computing.

H2: the type of relationship (i.e., anonymous users versus friends) moderates the effect of monetary incentives in a way that monetary incentives have a positive/negative effect on the willingness to share computational resources with anonymous users/friends.

H3: we propose a three way interaction between monetary incentive, type of relationship, and moral identity. More specifically, we hypothesize that in case of anonymous users/friends, monetary incentives will lead to a higher/lower motivation to share for participants with a high moral identity than for those with a low moral identity.

\subsection{Scenario Experiment}

We decided to test our framework in an online scenario experiment which we will report next. In this study, we asked participants to imagine a system in which they could share computational resources with other users. We experimentally manipulated whether they would receive a monetary reward or not and whether they would share their resources with anonymous private users or with their friends. We measured individual differences in centrality of moral identity using an established psychometric scale. We also included the questions from the preliminary study 


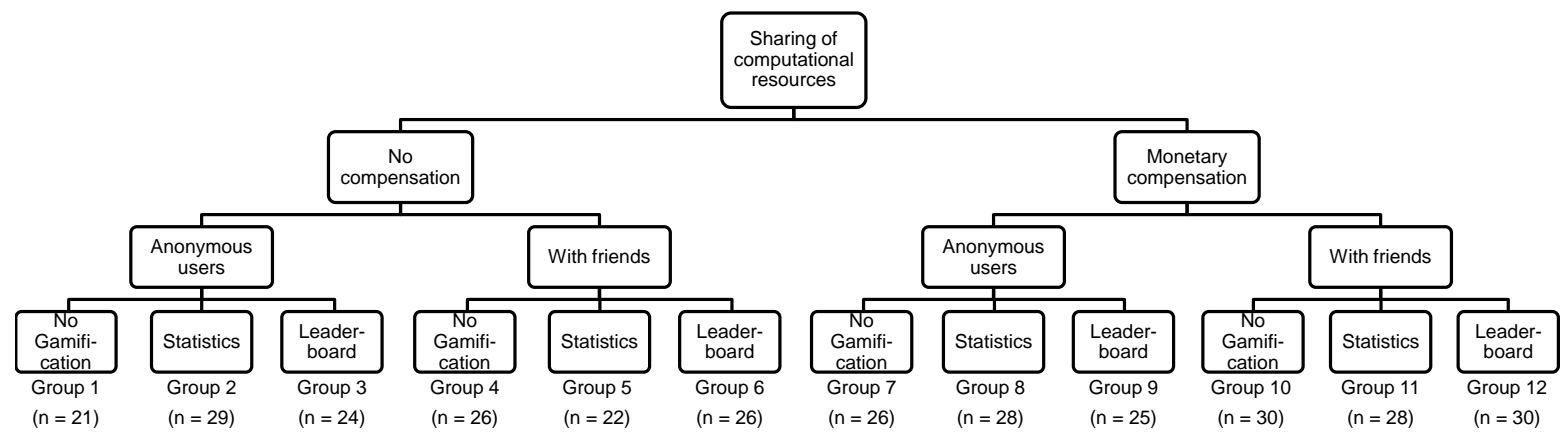

Figure 4. Design of the $2 \times 2 \times 3$ between subjects experiment.

to replicate the findings from the descriptive analysis with a larger sample (results are consistent but we do not report them for the sake of conciseness). Further, we decided to manipulate elements of gamification as this is a highly relevant question for the designers of such systems. Making the own and the contributions of other users transparent may enhance users' motivation and engagement due to gamification [15].

Design: We thus conducted a $2 x 2 x 3$ between subjects scenario experiment (i.e., no monetary compensation versus monetary compensation; sharing with anonymous users versus with friends; no gamification; statistics; leaderboard).

Procedure: We conducted the study as an online survey, programmed as a series of webpages. The invitation to participate in the study was sent out via Amazon's MTurk. Participants first read the scenario texts and then rated a set of Likert-type items (all ranging from 1 to 7). We measured participants' willingness to participate in such a system, manipulation checks, the questions from the pre-study, and moral identity (in this order). Finally, we elicited demographic information.

Treatment materials: We used the following treatment materials. All groups received an introduction which read as follows: 'Imagine a system that allows you to safely share computational resources.' As a manipulation for relationship type we added: 'Via this software package you can share computational resources with 1) friends; 2) anonymous users.' To manipulate the compensation we further explained: 'The sharing of resources is based on a system of 1) monetary compensation, i.e., you receive money for the computational resources which you share with other users in the system and you pay for the resources that you use or 2) reciprocity, i.e., you provide your computational resources to the other users in the system and may use their resources for free.' Last, as a manipulation gamification elements, we included three levels. In the 'no gamification' we added no further information. In the 'statistics' condition we explained: 'The software is further designed to transparently track your provision of resources. On a statistics page you can continuously track the amount of resources that you provided to other users and the amount of resources of others that you have used.' In the 'leaderboard' condition the text read as follows: 'The software is further designed as a game in which you compete with other users for the highest score. You can increase your score by providing resources to others. On a leaderboard you can see your performance compared to those of other users. Each day the best-performing contributor receives a symbolic award and earns extra points for the leaderboard.'

Measurement: To capture participants' willingness to participate, we included one straightforward item: 'How likely would you be to participate in the system?' with scale anchors ranging from ' $1=$ not likely at all' to '7=very likely'. To measure respondents' centrality of moral identity, we used four items from an established scale developed and tested by Aquino and Reed (2002). Participants are first provided with the following instructions: 'Listed below are some characteristics that might describe a person: Caring, compassionate, fair, friendly, generous, helpful, hardworking, honest, kind. The person with these characteristics could be you or it could be someone else. For a moment, visualize in your mind the kind of person who has these characteristics. Imagine how that person would think, feel, and act. When you have a clear image of what this person would be like, please report in how far you would agree with the following statements.' Then, they are asked to rate their agreement with the following items: 1 . 'It would make me feel good to be a person who has these characteristics.' 2. 'Being a person who has these characteristics is an important part of who I am.' 3. 'I am actively involved in activities that communicate to others that I have these characteristics.' 4. 'I strongly desire to have these characteristics.' The items were all rated on a seven point Likert scale ranging from 1 ('I do 
not agree at all') to 7 ('I fully agree'). The scale has a good internal consistency with a Cronbach's alpha value of .862 and all factor loading are above .649.

Sample: 498 US participants took part. During the testing of the questionnaire, the length of the survey was estimated to be approx. 15 minutes. In the MTurk sample, on average, it took participants only 10 minutes to answer the full questionnaire. We excluded those participants who answered the questionnaire in less than 8 minutes (183 individuals) because it is unlikely that they answered the questions attentively. As a consequence, we have a final sample of 315 valid responses. The mean age is 36.78 years and $47.9 \%$ of the respondents are female. The following table summarizes the exact sample sizes in all cells:

Manipulation checks: We included manipulation check items to make sure that our manipulations worked as intended. To test whether the manipulation of the social relationship (anonymous users versus friends) was effective, we included the following two items: 1) 'In the software package, I could share computational resources with various friends' and 2) 'In the software package, I could share computational resources with various anonymous users' with the scale anchors ' $1=I$ do not agree at all' and ' $7=$ I fully agree'. We used ANOVA to test for differences across groups. Using a dummy-coded variable (0 for anonymous users and 1 for friends) as an independent and the first item as a dependent variable we find a significant difference $\left(m_{\text {anonymoususers }}=4.66 ; m_{\text {friends }}=5.25 ; \mathrm{F}(1,315)\right.$ $=7.837, \mathrm{p}=.005)$. The same applies for the second item ( $m_{\text {anonymoususers }}=4.80 ; m_{\text {friends }}=3.54$; $\mathrm{F}(1,315)=29.174, \mathrm{p}=.000)$. To check whether respondents understood the monetary compensation manipulation, we included the following two items: 1) 'The sharing of resources in the software package is based on a system of monetary compensation, i.e., you receive money for the computational resources you share with the other users in the system and you pay for the resources that you use' and 'The sharing of resources in the software package is based on a system of reciprocity, i.e., you provide your computational resources to the other users in the system and you can use their resources for free' with the scale anchors ' $1=I$ do not agree at all' and ' $7=I$ fully agree'. Using a dummy-coded variable ( 0 for no monetary compensation and 1 for monetary compensation) as an independent and the first manipulation check item as a dependent variable we find a significant difference $\left(m_{\text {nocompensation }}=3.35 ; m_{\text {monetarycompensation }}=\right.$ $5.40 ; \mathrm{F}(1,315)=89.549, p=.000)$. This is also the case for the second item (mnocompensation = $4.95 ; m_{\text {monetarycompensation }}=3.13 ; F(1,315)=$
62.243, $p=.000)$. As a test of the third manipulation, i.e., elements of gamification, we included the following two items: 1) 'The software package is designed as a game in which you compete with other users for the highest score. You can increase your score by providing resources to others. On a leaderboard you can see your performance compared to those of other users. Each day the best-performing contributor receives a symbolic award and earns extra points for the leaderboard' ( $m_{\text {nogamification }}=2.93$; $m_{\text {statistics }}=3.03 ; m_{\text {leaderboard }}=5.45 ; F(2,315)=$ $55.091, p=.000) ; 2)$ 'The software package is designed to transparently track your provision of resources. On a statistics page you can continuously track the amount of resources that you provided to other users and the amount of resources of others that you have used' ( $m_{\text {nogamification }}=4.70 ; m_{\text {statistics }}=4.81$; $m_{\text {leaderboard }}=3.48 ; F(2,315)=15.120, p=$ $.000)$. Thus, the manipulation checks indicate that all manipulations worked as intended.

Analysis: To analyze our data, we first used ANOVA to check whether our treatments had significant effects on the central dependent variable, i.e., the willingness to participate in volunteer computing. In a second step, we computed moderated regression analyses using SPSS Process, model 3, to test for the hypothesized three-way interaction.

ANOVA results: In a first step, we ran ANOVAs to test for the effect of the treatments on willingness to participate. Supporting $H_{1}$, the monetary incentive significantly enhances willingness to participate (F $(1,315)=7.844, p=.005)$. Further, in line with $\mathrm{H}_{2}$, we find a significant two-way interaction between monetary incentive and type of relationship $(\mathrm{F}(1,315)$ $=3.985, \mathrm{p}=.045)$. Including a binary variable for moral identity centrality (MIC) $(0=$ low MIC, $1=$ high MIC, based on a median split, median $=5.5$ ), we find a significant three way interaction between monetary incentive, type of relationship, and $\mathrm{MIC}(\mathrm{F}(1,315)=$ $5.950, p=.015)$, providing first evidence for $H_{3}$. Figure 5 presents the means these groups.

Concerning the manipulation of gamification elements, we neither find direct nor interactive effects with the other treatments. Thus, we merely control for the manipulation in the analyses.

Moderated Regression Results: To formally test for the moderating role of moral identity centrality, we implemented a moderated regression analysis in SPSS process, model 3, with $\mathrm{Y}=$ willingness to participate, $\mathrm{X}=$ monetary incentive, $\mathrm{W}=$ relationship type friends, and $\mathrm{Z}=\mathrm{MIC}$. The results of the model estimation fully support our theorizing. The two-way interaction $(b=$ $4.35, p=.022$ ) as well as the three way interaction 

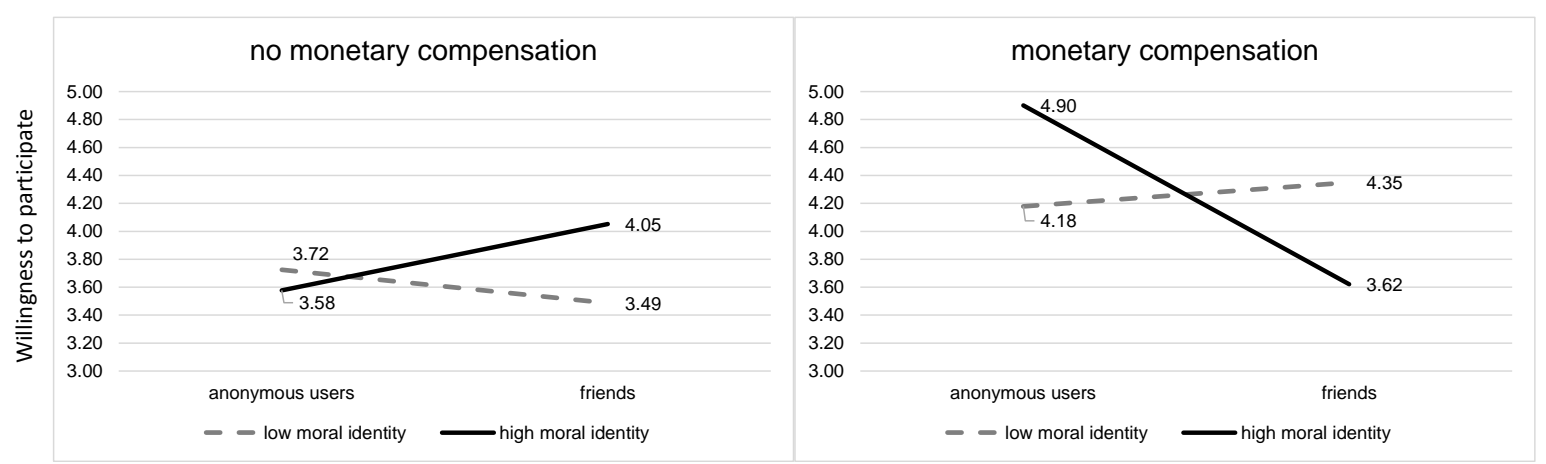

Figure 5. Willingness to participate in volunteer computing across groups.

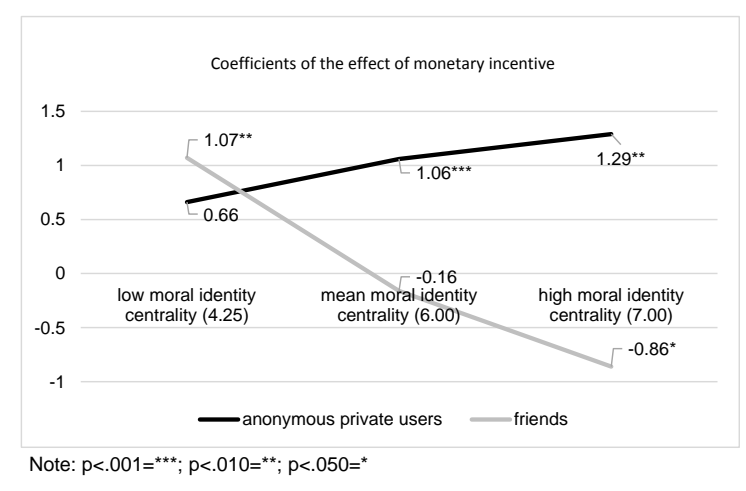

Figure 6. Simple slopes: the effect of monetary incentives on willingness to participate for different social relationships and varying levels of moral identity centrality.

$(b=-.93, p=.005)$ are significant. Further, simple slopes analyses reveal interesting insight about the effect of the independent variable monetary incentive on the dependent variable of willingness to participate for different levels of the moderators.

Figure 6 graphically illustrates these results. In case of anonymous users, the effect of monetary incentives is insignificant for lower levels of MIC and significantly positive for mean and higher levels. For the case of sharing with friends, in contrast, the effect of monetary incentives is significantly positive for lower levels of MIC, insignificant for mean levels, and significantly negative for higher levels. Whereas the coefficients of the effect of monetary incentives on willingness to participate for anonymous private users are not significantly different from each other, the coefficients for friends do differ significantly indicated by confidence intervals which do not overlap $\left(b_{\text {low } M I C}=1.07,[.3102 ; 1.8235] ; b_{\text {highMIC }}=\right.$ $.086,[1.6906 ;-.0282]$.

\section{Discussion}

Our results have implications for academic theory and for the design of platforms for sharing of computational resources alike. To the best of our knowledge, this paper is the first which combines the two theories of relational models and motivation crowding to explain sharing behavior. While P2P computing is only one application area among multiple examples, further research is necessary to validate these results in other parts of the sharing economy where sharing happens among strangers and friends. In terms of conceptual contributions, our results contribute to four literature streams. First, we contribute to the literature on $\mathrm{P} 2 \mathrm{P}$ platforms in the sharing economy. The results of our scenario experiment indicate that monetary incentives may have detrimental effects on participation of the type of relationship between users is a social relationship and thereby tie in very well with recent findings on social versus market-based exchanges in the sharing economy. Second, our study links to the evolving stream of research on relational models in general by providing another application in which these models play a decisive role [25]. Third, only very few papers have so far scrutinized the factors that moderate the effect of extrinsic incentives on prosocial behaviors. While conceptual work has laid out the mechanisms by which crowding-in and crowding-out effects occur, only very little empirical research has so far identified and empirically tested moderating variables that explain this "flip" (e.g., [26]). Fourth, by testing moral identity centrality as a moderator in our model, we contribute to the incipient research stream that proposes this construct to be an important determinant of prosocial behaviors [21]. In terms of practical implications, our results suggest that software developers attempting to design tools for the sharing of computational resources should take into account that monetary incentives can enhance or harm willingness to participate depending on social 
relationships and personal characteristics. As every study, this study has limitations which at the same time represent avenues for future research. First, our scenario experiment relies on an intentional measure as dependent variable. It is widely known that intentions and behaviors may differ and it would be a necessary next step to replicate this study's results in a setting that includes objectively measured outcomes. This could be done in a field-experiment with a real application of P2P resource sharing. This would also allow for the analysis of more diverse behavioral dependent variables. We expect gamification to show an effect when applied in a field study. As gamification leverages peoples' emotions and desires it can hardly be simulated in scenario experiments but reveals its full potential only in real world settings. Second, we only test for the effect of monetary rewards versus no monetary rewards while the spectrum of possible incentives is much broader. A follow-up study could test various incentives ranging from more intrinsic to more extrinsic rewards and observe the respective motivation crowding-effects. Finally, while we chose to focus on moral identity centrality, there could be other important personality factors that we neglected. Future research could set out to test other possible individual-level factors that capture unexplained variance.

\section{References}

[1] J. Hamari, M. Sjöklint, and A. Ukkonen, "The sharing economy: Why people participate in collaborative consumption," Journal of the Association for Information Science and Technology, vol. 67, no. 9, pp. 2047-2059, 2016.

[2] M. J. Litzkow, M. Livny, and M. W. Mutka, "Condor - a hunter of idle workstations," Proceedings of the 8th Int. Conf. on Dist. Computing Systems, pp. 104-111, 1988.

[3] G. Woltman and S. Kurowski, "The great internet mersenne prime search," URL: http://www.mersenne.org/(visited on 05/30/2018).

[4] D. P. Anderson, "BOINC: A system for public-resource computing and storage," Proceedings - IEEE/ACM Int. Workshop on Grid Computing, pp. 4-10, 2004.

[5] C. Haas, S. Caton, and C. Weinhardt, "Engineering incentives in social clouds," in Proceedings of the 2011 11th IEEE/ACM International Symposium on Cluster, Cloud and Grid Computing, pp. 572-575, IEEE Computer Society, 2011.

[6] A. P. Fiske, "The four elementary forms of sociality: framework for a unified theory of social relations.," Psychological review, vol. 99, no. 4, p. 689, 1992.

[7] J. Heyman and D. Ariely, "Effort for payment: A tale of two markets," Psychological science, vol. 15, no. 11, pp. 787-793, 2004.

[8] B. S. Frey and F. Oberholzer-Gee, "The cost of price incentives: An empirical analysis of motivation crowding-out," The American economic review, vol. 87, no. 4, pp. 746-755, 1997.
[9] B. S. Frey and R. Jegen, "Motivation crowding theory," Journal of economic surveys, vol. 15, no. 5, pp. 589-611, 2001.

[10] M. Mowbray, "Ourgrid: a web-based community grid," in Web Based Communities, p. 347, 2006.

[11] K. Chard, S. Caton, O. Rana, and K. Bubendorfer, "Social cloud: Cloud computing in social networks," in Cloud Computing (CLOUD), 2010 IEEE 3rd International Conference on, pp. 99-106, IEEE, 2010.

[12] K. Chard, K. Bubendorfer, S. Caton, and O. F. Rana, "Social cloud computing: A vision for socially motivated resource sharing," IEEE Transactions on Services Computing, vol. 5, no. 4, pp. 551-563, 2012.

[13] V. D. Cunsolo, S. Distefano, A. Puliafito, and M. Scarpa, "Volunteer computing and desktop cloud: The cloud@ home paradigm," in Network Computing and Applications, 2009. NCA 2009. Eighth IEEE International Symposium on, pp. 134-139, IEEE, 2009.

[14] R. M. Ryan and E. L. Deci, "Intrinsic and Extrinsic Motivations: Classic Definitions and New Directions," Contemporary Educational Psychology, vol. 25, pp. $54-67,2000$.

[15] S. Deterding, D. Dixon, R. Khaled, and L. Nacke, "From game design elements to gamefulness: defining gamification," in Proceedings of the 15th international academic MindTrek conference: Envisioning future media environments, pp. 9-15, ACM, 2011.

[16] D. Ariely, A. Bracha, and S. Meier, "Doing good or doing well? image motivation and monetary incentives in behaving prosocially," American Economic Review, vol. 99, no. 1, pp. 544-55, 2009.

[17] K. Chard, K. Bubendorfer, S. Caton, and O. F. Rana, "Social cloud computing: A vision for socially motivated resource sharing," IEEE Transactions on Services Computing, vol. 5, no. 4, pp. 551-563, 2012.

[18] M. Conti and M. Kumar, "Opportunities in opportunistic computing," Computer, vol. 43, no. 1, 2010.

[19] R. Bénabou and J. Tirole, "Incentives and prosocial behavior," American economic review, vol. 96, no. 5, pp. 1652-1678, 2006.

[20] K. Aquino, I. Reed, et al., "The self-importance of moral identity.," Journal of personality and social psychology, vol. 83, no. 6, p. 1423, 2002.

[21] K. Aquino, D. Freeman, A. Reed II, V. K. Lim, and W. Felps, "Testing a social-cognitive model of moral behavior: the interactive influence of situations and moral identity centrality.," Journal of personality and social psychology, vol. 97, no. 1, p. 123, 2009.

[22] K. Aquino, A. Reed, M. M. Stewart, and D. L. Shapiro, "Self-regulatory identity theory and reactions toward fairness enhancing organizational policies," What motivates fairness in organizations, pp. 129-148, 2005.

[23] D. K. Lapsley and D. Narvaez, "Moral development, self and identity: Essays in honor of augusto blasi," 2004.

[24] A. Blasi, "Moral functioning: Moral understanding and personality," Moral development, self, and identity, pp. 335-347, 2004.

[25] S. A. Haslam, Psychology in organizations. Sage, 2004.

[26] L. M. Edinger-Schons, J. Sipilä, S. Sen, G. Mende, and J. Wieseke, "Are two reasons better than one? the role of appeal type in consumer responses to sustainable products," Journal of Consumer Psychology, 2018. 